\title{
STRUCTURE OF LARGE NONCRYSTALLINE LENNARD-JONES MODELS
}

\author{
Yu. I. Naberukhin and V. P. Voloshin
}

UDC 532.74

The structure of computer models (100,000 atoms interacting with the Lennard-Jones potential) is analyzed for liquids and amorphous solids at different temperatures. The structural laws of instantaneous and inherent $(I$ and $F$ ) structures are compared. Ample information about structural regularities may be obtained using Delaunay simplices (DS) as structural elements. Various distributions of the properties of the simplices are considered which allow one to determine the prevailing forms of simplices and their transformations at different temperatures. Percolation analysis is used to describe the spatial arrangement of simplices throughout the model.

Keywords: simple liquids, structure of a liquid, computer simulation.

\section{INTRODUCTION}

The idea that the structure of liquids and amorphous solids is characterized by only short-range order has long been outdated. Disordered close packing of atoms is not totally chaotic, but has regularities extending through many interatomic distances, sometimes called medium-range order [1]. It is now also clear that these laws cannot be formulated based on the crystal order. Ziman's opinion that "disorder [in liquids] is not mere chaos; it implies defective [crystal] order" [2] is certainly wrong. Bernal repeatedly emphasized that the arrangement of liquids has nothing in common with the crystal structure, but should be characterized by specific laws [3,4]. To reveal these laws, special methods of investigation other than radial distribution functions, generally employed for structure description, are needed. An approach based on the Voronoi-Delaunay geometrical methods provides an effective tool of investigation [5]. One of the key notions of this method - the Voronoi polyhedron - was introduced into general use by Bernal. Another notion, the Delaunay simplex, was regularly used in our works [6-13] and proved even more useful for describing the structure of simple liquids.

Structure description is generally a two-step procedure. Structural elements are to be chosen, and the laws of their arrangement in space are to be revealed. If these laws are expressed in terms of radial distribution functions, then an individual atom is implied to be a structural element. We use an approach where a quadruple of neighboring atoms are chosen as a basic structural element. More precisely, these four atoms must be geometric neighbors; i.e., their Voronoi polyhedra must have common faces. The centers of these four atoms define the vertices of a tetrahedron called a Delaunay simplex (DS) $[5,7]$. Delaunay simplices are unequivocally defined by the configuration of the system, i.e., by the coordinates of the centers of all its atoms. They fill space without gaps or overlaps, which results in three-dimensional triangulation (tessellation) of space occupied by the system. The choice of Delaunay simplices as structural elements is fruitful because dense monoatomic liquids have two prevailing types of simplex close in form to the ideal tetrahedron and quartoctahedron (a quarter of a perfect

Institute of Chemical Kinetics and Combustion, Siberian Division, Russian Academy of Sciences, Novosibirsk; naber@kinetics.nsc.ru. Translated from Zhurnal Strukturnoi Khimii, Vol. 47, Supplement, pp. S129-S143. Original article submitted April 12, 2006. 
octahedron) called good tetrahedron and good quartoctahedron [6-13]. As is known [14], the crystal structure of close packings of spheres may be represented as a set of perfect tetrahedra and octahedra (that define the configuration of interatomic voids). Thus the structural elements that prevail in the structure of a liquid (Delaunay simplices) are the same as those that form the structure of a crystal. In crystal, however, the relative arrangement of these elements is absolutely different. While crystals always have quartoctahedra united into octahedra, liquids primarily have individual quartoctahedra, sometimes integrated into semioctahedra, but very seldom into whole octahedra.

The advantage of this choice of structural elements is that their relative positions can be investigated by methods of percolation theory $[15,16]$. The centers of Delaunay simplices (the centers of circumspheres around their vertices) are the sites of the Voronoi network, which is a set of vertices and edges of a mosaic composed of all Voronoi polyhedra of the system. Percolation analysis is selecting ("coloring") the sites on this network that correspond to simplices with certain metrical properties (for example, good tetrahedra or quartoctahedra) and considering their relative positions (contiguity). Importantly, one can proceed with arbitrarily distant simplices since the Voronoi network is defined throughout the model. While investigation of the properties of the individual Voronoi polyhedra or Delaunay simplices (or of the radial distribution functions of atoms) provides information about the local structure, percolation analysis allows us to consider the structure of the model as a whole, i.e., the total structure (the term introduced by P. M. Zorky [17, 18]). As shown in our previous works $[5,6,8]$, the total structure of a simple liquid involves Delaunay simplices (nearly ideal tetrahedra, which are the majority of all DS) arranged into long branched chains with built-in decahedra - rings of five edge-sharing tetrahedra.

The stated structural regularities of simple liquids were obtained on small models of 108 [6-9], 500 [10, 12], and 8000 [11] particles interacting with the Lennard-Jones potential. The purpose of the present work is to confirm and refine these laws using larger models (100,000 atoms) corresponding to different thermodynamic conditions. Large models have many advantages. They allow better averaging of statistical noise. The radial distribution functions of these models may be traced to distances of more than 20 particle diameters after which oscillations die away; this enables correct calculation of the structure factor. Finally, under certain thermodynamic conditions, the atomic packing becomes nonuniform, acquiring voids of more than ten atomic diameters; clearly, these situations cannot be investigated with small models. By varying the density and temperature of model preparation, one can observe interesting effects of structure transformation. Earlier [13] we investigated the structure of empty space and the motions of test particles in these models.

\section{COMPUTER MODELS}

Each model contained 100,000 atoms interacting with the Lennard-Jones potential $V_{\mathrm{LJ}}(r)=4 \varepsilon\left[(\sigma / r)^{12}-(\sigma / r)^{6}\right]$ in a cubic cell with periodic boundary conditions for three values of density: $\rho^{*}=0.7,0.85,0.9$ and nine values of temperature: $T^{*}$ from 0.8 to 0 (at a step of 0.1 ). We use reduced values of density and temperature everywhere: $\rho^{*}=\sigma^{3} \rho, T^{*}=k T / \varepsilon$, where $\rho=N / V$ is particle density. The atomic diameter, i.e., the position of the potential minimum $d=2^{1 / 6} \sigma$ is chosen for the length unit.

The models were prepared by the following procedure. For each value of density, a random rarefied packing of atoms was generated and then compressed by simply scaling the coordinates to achieve the required density. Then the highest temperature $\left(T^{*}=0.8\right)$ was set in the model, and the system was relaxed in an NVT ensemble by the Monte Carlo method. The temperature was subsequently lowered by 0.1 , and the system relaxed to obtain the next configuration. At each stage, relaxation was repeated until the average reduction of energy per cycle (one step of the Monte Carlo procedure for each atom) reached $10^{-6}$ and variation of pressure after 1000 cycles became appreciably smaller than the amplitude of oscillations within the same time interval. As a result, each stage was from 2000 high-temperature cycles to 100,000 low-temperature cycles.

This article discusses only the properties of models with $\rho^{*}=0.85$, while models with other densities are mentioned simply for the sake of comparison. This density equals the density at the triple point for the Lennard-Jones system: $\rho_{t}{ }^{*}=$ $0.85 \pm 0.01$ and $T_{t}^{*}=0.68 \pm 0.02$ [19]. Therefore at $T^{*}=0.8$ and 0.7 our models correspond to an equilibrium liquid, while at 

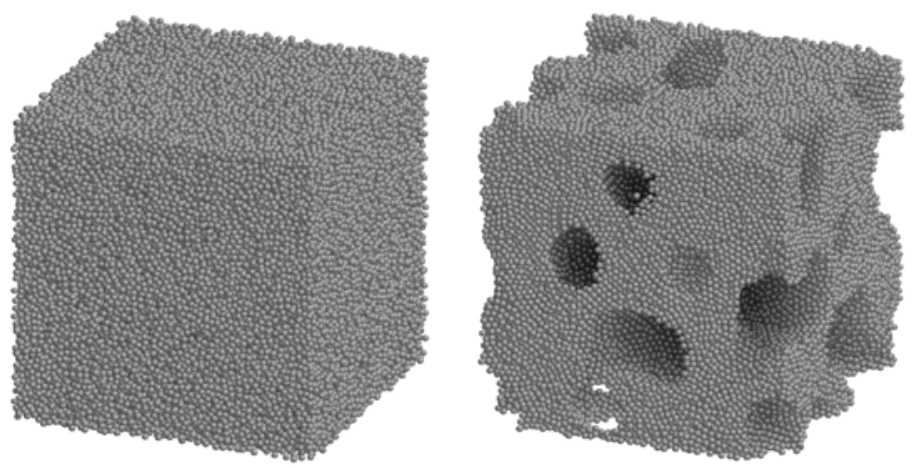

Fig. 1. General view of the model of 100,000 particles for density $\rho^{*}=0.7$ and temperatures $T^{*}=0.8$ (left) and $T^{*}=0.1$ (right).

lower temperatures these are either overcooled liquids or unstable solids. The latter can occur in two states. At high temperatures, they form uniform phases where particles experience tension stress, resulting in negative pressures. At reduced temperatures, these tensions often lead to loss of uniformity: large cavities appear along with regions of nontensioned close packing of atoms. This situation is already visualized on models. Figure 1 shows the general view of two models. Based on similar images of all our models one can conclude that at the highest temperature $\left(T^{*}=0.8\right)$ all models are uniform, with atoms distributed almost uniformly throughout the model, as shown Fig. 1 (left). Loss of uniformity is easily recognized from the occurrence of large cavities, as shown in Fig. 1 (right). When density is 0.7, cavities of this kind appear already at a temperature of 0.7. As density increases to 0.85 , the temperature at which the cavities form decreases to 0.3 . In packings with the highest density $\left(\rho^{*}=0.9\right)$, cavities do not appear at all, and the model remains uniform at any temperature. (The phase transition of the loss of uniformity was investigated earlier on models of 8000 atoms [11].) The void diameter often exceeds 10 particle diameters. Thus nonuniform systems cannot be studied on small models of $\sim 1000$ particles (generally used in computer simulation). That is why large models are preferable.

$\boldsymbol{F}$ structure. For seeking quantitative structural laws in disordered systems, it is necessary to remove thermal noise. The structure of a crystal (in the conventional sense) is defined by the positions of the local minima of potential energy, but never by the instant positions of its atoms, which are always disordered and do not possess translation symmetry. Similarly, the structure of a liquid should be defined by the positions of local minima on the potential energy hypersurface. The positions of these minima specify the so-called hidden [20] or inherent (proper) structure of a liquid, which differs strongly from the instantaneous structure ( $I$ structure) specified by the instant positions of atoms. The positions of the local minima may be found by using steepest descent methods along the gradient of the potential [20-23]. For our purposes, however, it is quite enough to eliminate only basic thermal chaos. This can easily be done by relaxing the given instantaneous structure using the Monte Carlo method at $0 \mathrm{~K}$ and constant density; for this, all particles are randomly shifted toward the local energy minimum. The resulting structure is called an $F$ (frozen) structure [24, 25]. Figure 2 shows the typical curve of energy and pressure evolution from $I$ to $F$ structure. We can see that thermal chaos is basically removed after fifty steps followed by slow drifting of the model toward the local minimum. All our $F$ structures were obtained after 500 steps of relaxation and did not demand high computing times. In $F$ structures, pressures are always negative.

\section{PAIR RADIAL FUNCTIONS AND STRUCTURE FACTORS}

The radial distribution functions are shown in Figs. 3 and 4. We generally prefer to represent difference radial functions modified by multiplication by $r^{2}, G(r)=4 \pi \rho r^{2}[g(r)-1]$, instead of the pair correlation functions $g(r)$, which are rapidly damping functions. Long-range oscillations are well visible here even at distances $r>10 d$. In addition to the standard high-frequency oscillations caused by correlations in atomic arrangement, there are low-frequency oscillations at $T^{*} \leq 0.3$ 


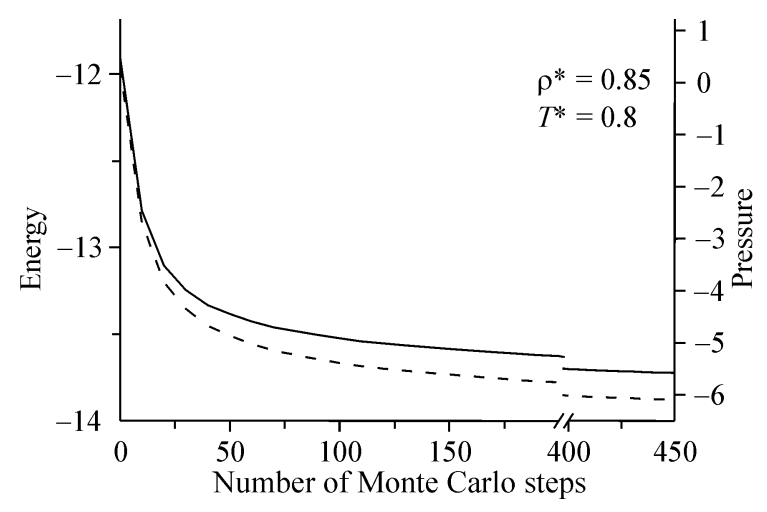

Fig. 2. Evolution of energy (solid line) and pressure (dashed line) during a transition from $I$ to $F$ structure.
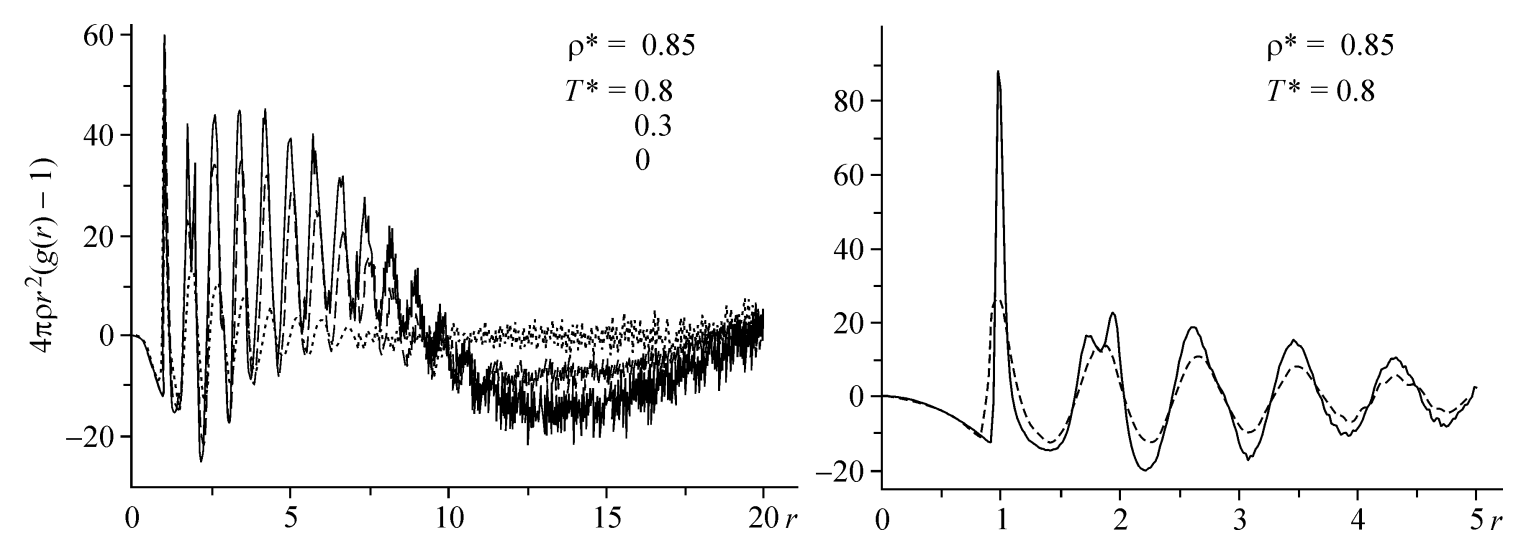

Fig. 3. Modified difference radial distribution functions. Left: $I$ structure. Right: comparison of $F$ (solid line) and $I$ (dotted line) structures at $T^{*}=0.8$.
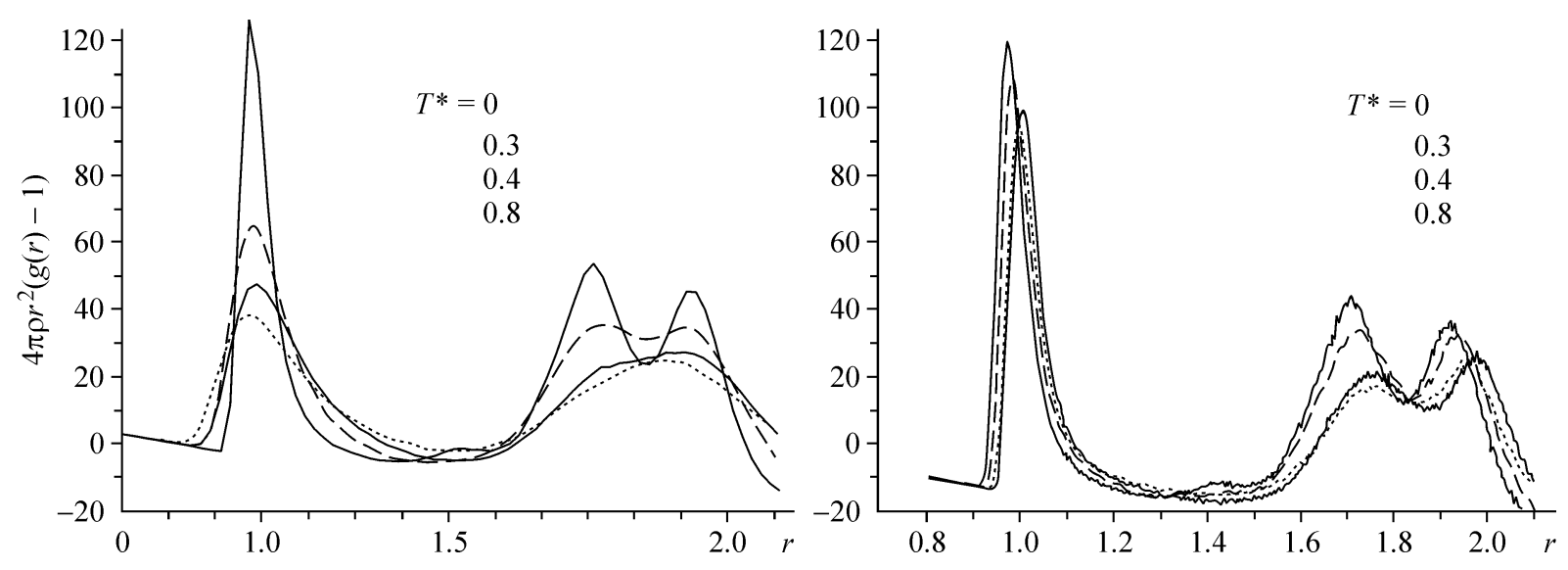

Fig. 4. First maxima of the radial distribution functions for $I$ (left) and $F$ (right) structures. $\rho^{*}=0.85$.

(20d equals approximately one oscillation period; Fig. 3, left). They obviously reflect correlations in the arrangement of large cavities, which appear at these temperatures (they are practically indiscernible on the $g(r)$ curve). These oscillations can serve as a good criterion for the loss of uniformity in the model.

Figure 3 (right) shows the interrelation between the radial functions of $I$ and $F$ structures. First, it can be seen that the oscillation phases coincide in the two functions, demonstrating that the packing principles are identical for the $I$ and $F$ 

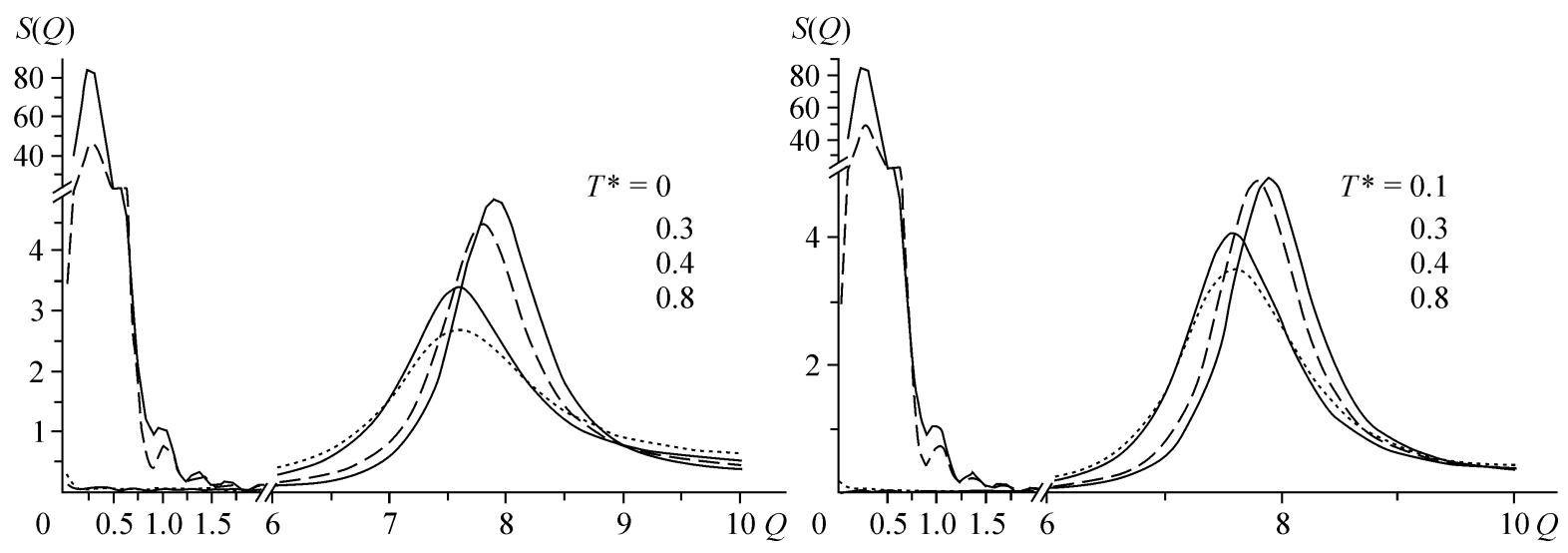

Fig. 5. First peaks of the structure factor: $I$ structure (left) and $F$ structure (right). $\rho^{*}=0.85$.

structures, just as they are expected to be. Second, for the $F$ structure, the second peak is split into two subpeaks. The doublet structure of the second peak is generally believed to be a characteristic feature of the amorphous state, reflecting the difference between its structure and the structure of liquids (in which case the doublet is absent) [26]. Figure 3 shows that this is a wrong opinion. In liquids, the characteristic properties of packing are indistinct in the $I$ structure because of thermal chaos; in the $F$ structure, however, they exist in the latent state even at high temperatures $\left(T^{*}=0.8\right)$. The sense of this effect is quite clear: as shown earlier [10], discrete subpeaks are caused by chains of good tetrahedra and quartoctahedra, which are the basic structural elements of a liquid. Therefore the doublet structure of the second peak persists in $F$ structures at all temperatures (Fig. 4, right).

The $G(r)$ functions may be divided into two groups corresponding to uniform (at $T^{*} \geq 0.4$ ) and nonuniform packing (at $T^{*} \leq 0.3$ ) (Fig. 4). When the temperature decreases, uniformity is lost between $T^{*}=0.4$ and $T^{*}=0.3$; then internal tensions are removed, and the distance between the nearest particles decreases sharply by approximately $0.025 d$ (Fig. 4, right). At $T^{*} \leq 0.2$, a weak peak appears at $r \approx \sqrt{2}$, which corresponds to the radius of the second coordination sphere in fcc and hcp crystals. This peak indicates that the system has small regions with nearly crystalline packing of atoms.

Pictures of the structure factor can also be divided into two groups (Fig. 5). At $T^{*} \leq 0.3$ there is a strong peak at small values of the wave vector $Q_{\mathrm{M}} \approx 0.25$ corresponding to diffraction on large cavities. At $T^{*} \geq 0.4$ this peak is absent, which is evidence of the uniformity of the system. The position of the fundamental peak, $Q_{1}$, is independent of temperature in a uniform system, indicating that the principle of atomic packing did not change. Because $Q_{1} / Q_{\mathrm{M}} \approx 30$, the average distance between large cavities in a nonuniform system is approximately 30 atomic diameters.

\section{CHARACTERISTICS OF THE INDIVIDUAL VORONOI POLYHEDRA AND DELAUNAY SIMPLICES}

Of many properties of Voronoi polyhedra (VP) we consider here only their volume, which is the inverse local density at the site of the given atom. Volume distributions of VP are shown in Fig. 6. In uniform models, they are centered near the average value of VP volume: $\left\langle V_{\mathrm{VP}}\right\rangle=\left(\sqrt{2} \rho^{*}\right)^{-1}=0.832$. In $F$ structures, the distribution width is almost independent of temperature (as it must be according to its sense), whereas in $I$ structures, the width slightly increases with temperature. When cavities appear $\left(T^{*} \leq 0.3\right)$, the maximum of distributions lies at considerably smaller values than the average volume. This means that distributions have on their right slope a long weak tail not visible on the scale of the figure. Indeed, nonuniform models occasionally have VP whose volume is $>80$, i.e., two orders of magnitude larger than the average volume. They obviously correspond to atoms lying near the borders of cavities; the volume of VP for these atoms includes some part of their volume. 


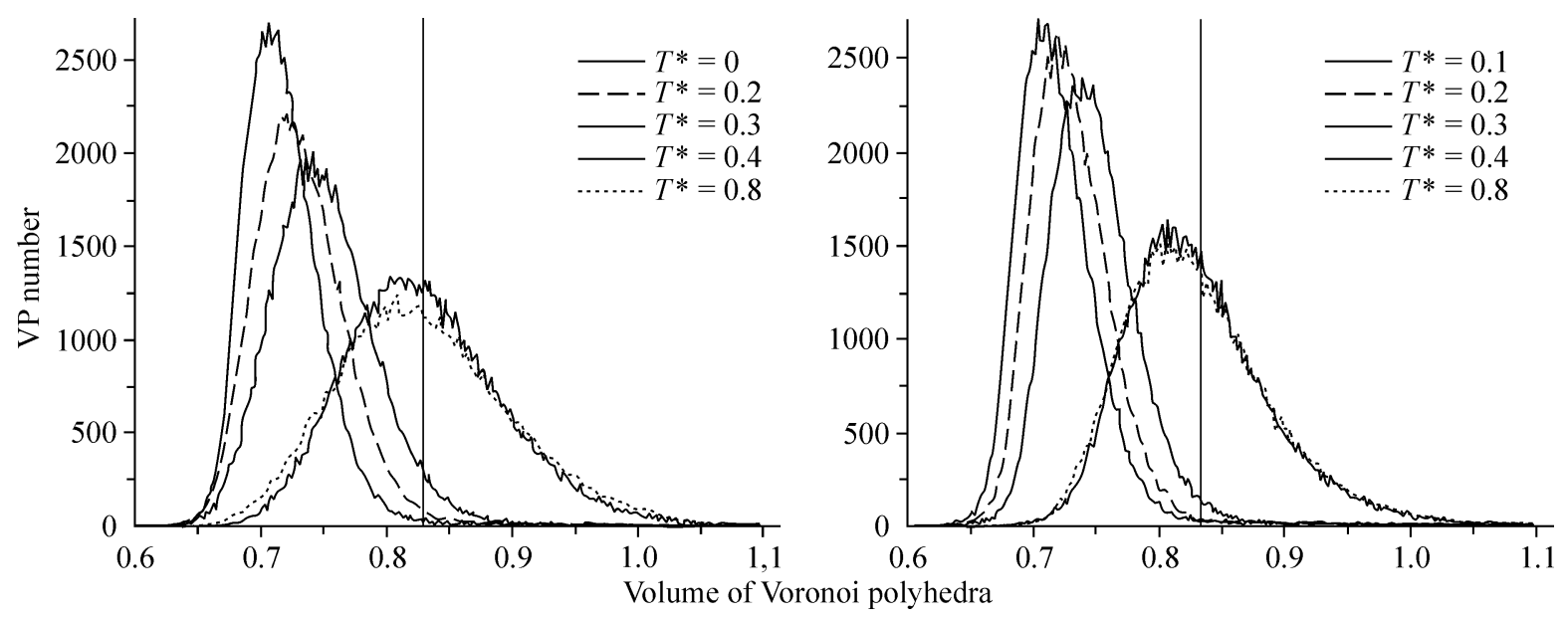

Fig. 6. Volume distribution of the Voronoi polyhedra: $I$ structures (left) and $F$ structures (right). $\rho^{*}=0.85$. The vertical line shows the average value of volume.

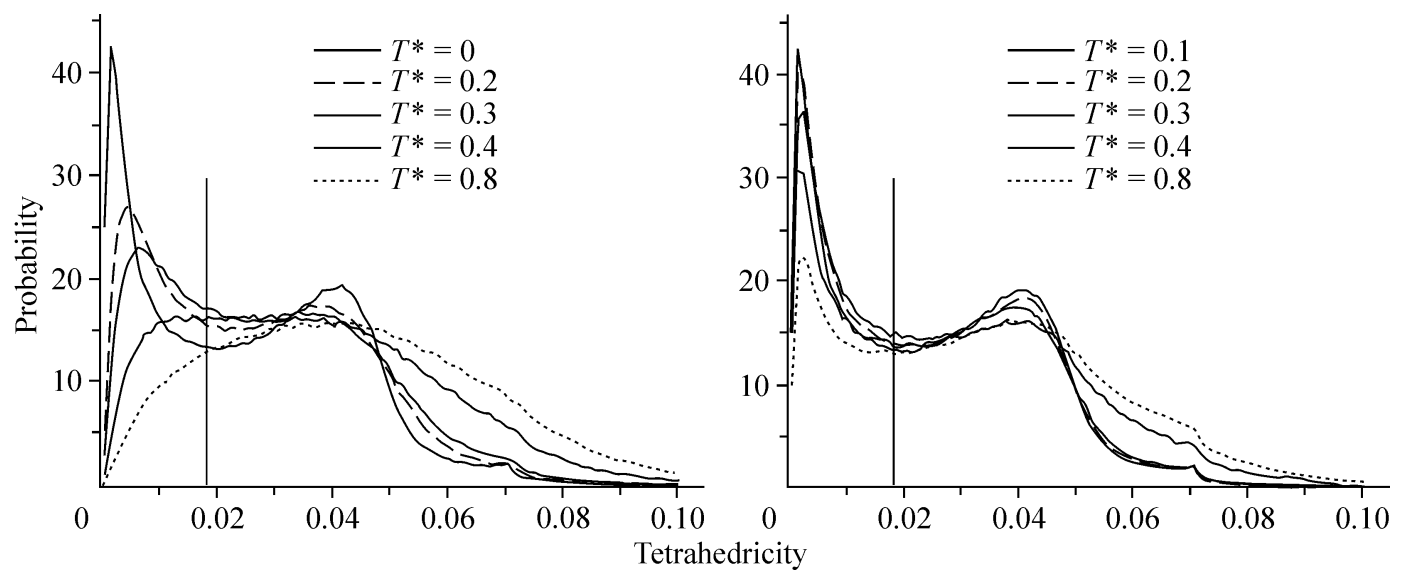

Fig. 7. Tetrahedricity distribution of Delaunay simplices: $I$ structures (left) and $F$ structures (right). $\rho^{*}=0.85$. The vertical line shows the boundary of good tetrahedra $T_{\mathrm{B}}=0.018$.

To characterize the form of Delaunay simplices (DS) we use, as before [6-8], the tetrahedricity index

$$
T=\sum_{i>j}\left(l_{i}-l_{j}\right)^{2} /\left(15\langle l\rangle^{2}\right)
$$

and also the octahedricity index

$$
O=\sum_{\substack{i>j \\ i, j \neq m}}\left(l_{i}-l_{j}\right)^{2} /\left(10\langle l\rangle^{2}+\sum_{i \neq m}\left(l_{i}-l_{m} / \sqrt{2}\right)^{2} /\left(5\langle l\rangle^{2}\right),\right.
$$

where $l_{i}$ is the edge length of the DS; $l_{m}$ is the length of the longest edge; $\langle l\rangle$ is the average length of six edges of the simplex. In the ideal tetrahedron, $T=0$, and in the ideal quartoctahedron (i.e., in a quarter of the ideal octahedron), $O=0$. The distributions of these characteristics are shown in Figs. 7 and 8. The number of good tetrahedra and quartoctahedra can be seen to increase drastically from $I$ to $F$ structure. Indeed, elimination of thermal chaos reduces the edge length fluctuations of DS and reveals the true regularities of the structure. The presence of sharp peaks at low values of $T$ and $O$ in $F$ structures unambiguously shows that good tetrahedra and quartoctahedra are the preferred types of simplex. At low temperatures (Fig. 7) the tetrahedricity distribution curve contains a weak peak at $T \approx 0.07$, which corresponds to a DS called Kije simplex (see [7] and [5], pp. 117/118). It has the form of a slightly distorted square with nearly zero volume and with two diagonals not lying in one plane. A Kije simplex is formed by weak distortion of the octahedral configuration of atoms [7]; its presence indicates that some quartoctahedra are united into full octahedra. 


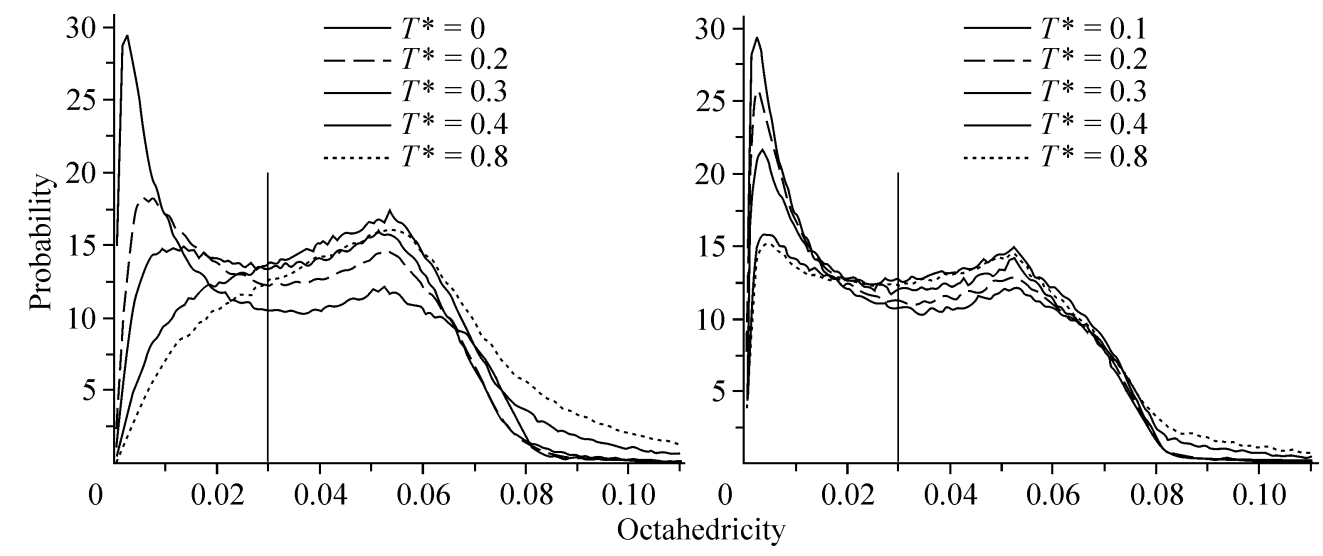

Fig. 8. Octahedricity distribution of Delaunay simplices: $I$ structures (left) and $F$ structures (right). $\rho^{*}=0.85$. The vertical line shows the boundary of good octahedra $O_{\mathrm{B}}=0.03$.
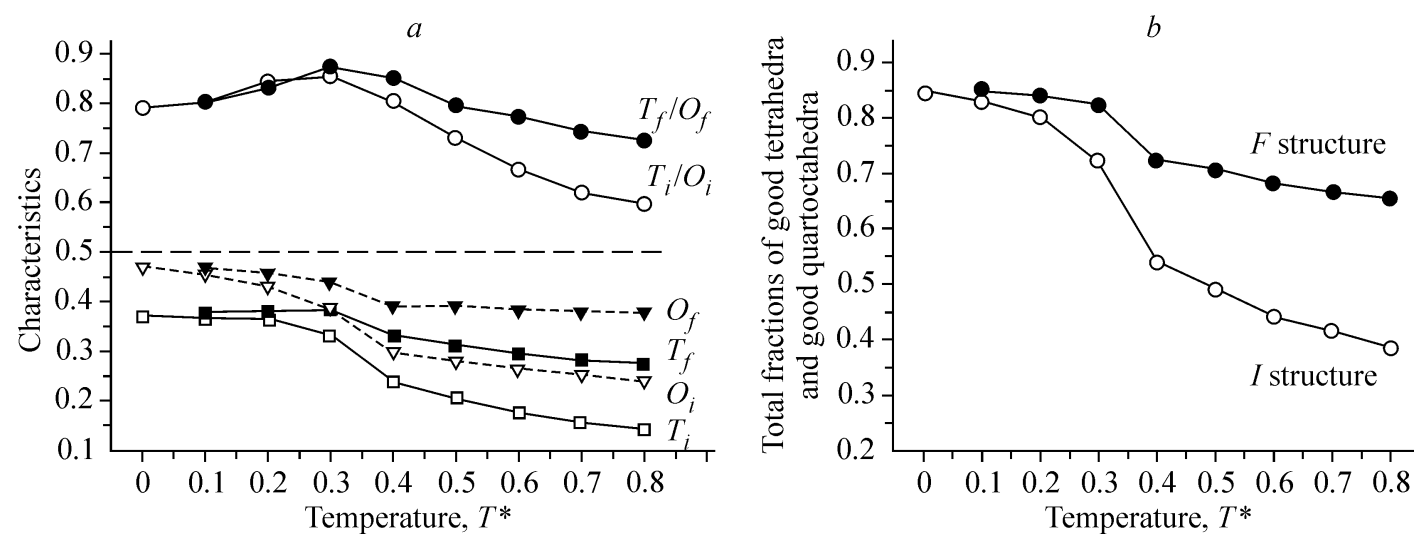

Fig. 9. Temperature behavior of fractions of good tetrahedra (squares), good quartoctahedra (triangles) and their ratios (circles). Empty symbols: $I$ structure, full symbols: $F$ structure $(a)$. Temperature dependence of the total fractions of good tetrahedra and quartoctahedra $(b)$.

As previously (see [5], pp. 119-121), we choose $T_{\mathrm{B}}=0.018$ as the boundary of good tetrahedra, and $O_{\mathrm{B}}=0.03$ as the boundary of good quartoctahedra. Then, by integrating the corresponding distributions from zero to these boundaries we obtain fractions of good tetrahedra and quartoctahedra shown in Fig. 9a. All these fractions grow with decreasing temperature, but do not reach the crystal values even at the lowest temperature. At $T=0$ the fraction of good tetrahedra exceeds the crystal value $1 / 3$, while the fraction of good quartoctahedra is much smaller than $2 / 3$. Therefore the ratio of the number of good tetrahedra to the number of good quartoctahedra is significantly higher than the crystal value $1 / 2$ at all temperatures. This is a characteristic regularity of the structure of simple liquids, indicating that their arrangement differs dramatically from that of crystals.

Good tetrahedra and good quartoctahedra together represent a significant part of all Delaunay simplices in the system (Fig. $9 b$ ). Even at the highest temperature (0.8), their fraction is approximately $40 \%$ in the $I$ structure and more than $70 \%$ in the $F$ structure. Thus these types of Delaunay simplices are really the basic structural elements of the disordered dense states of Lennard-Jones systems.

The radius of a sphere around four vertices of the DS is also a useful characteristic of this figure. The radius distributions (Fig. 10) are classed into distributions for uniform and nonuniform models. For $F$ structures, the distributions are bimodal, which reflects the presence of two sorts of simplex: good tetrahedra and quartoctahedra. In nonuniform models $\left(T^{*} \leq 0.3\right)$ where tensile stresses are removed, the characteristics of tetrahedra are close to optimal (crystalline) when atoms occupy the minima of the pair potential. 


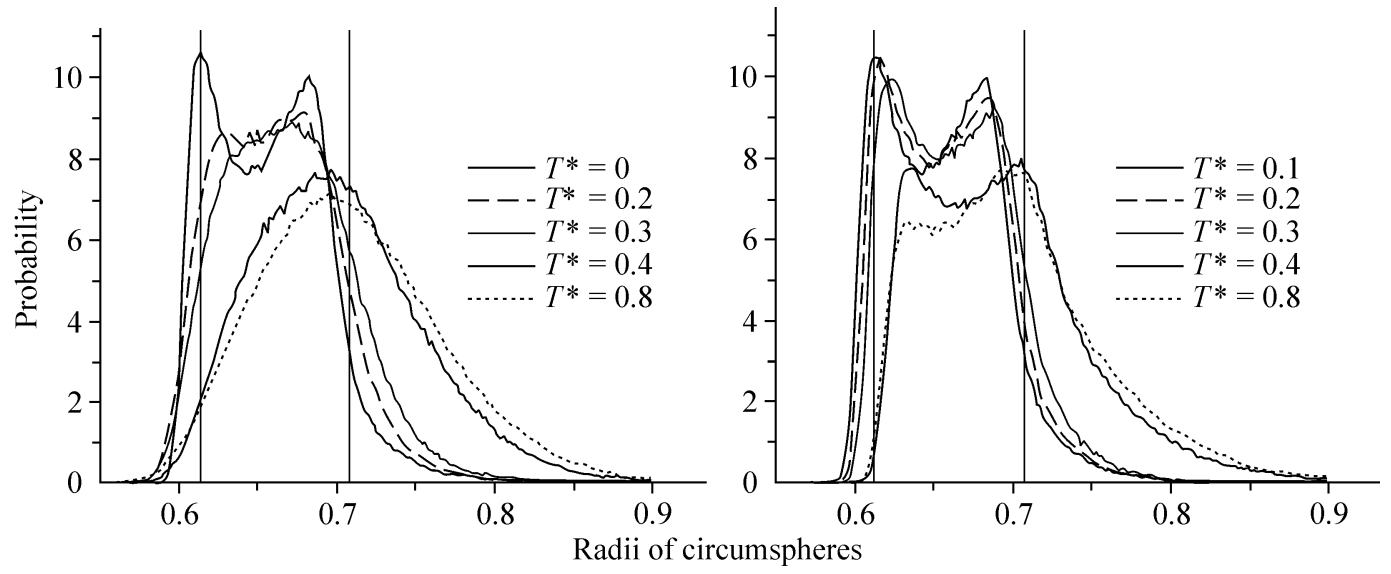

Fig. 10. Distribution of the radii of the circumspheres around Delaunay simplices: $I$ structures (left) and $F$ structures (right). $\rho^{*}=0.85$. Vertical lines show the circumsphere radii around the ideal tetrahedron $(R=0.612)$ and ideal quartoctahedron $(R=0.707)$.

\section{JOINT DISTRIBUTIONS OF CHARACTERISTICS OF DELAUNAY SIMPLICES}

Details on the structure of a system may be obtained by considering joint distributions of any two characteristics of Delaunay simplices. Figures 11 and 12 show the joint distributions of tetrahedricity and octahedricity for two temperatures. The distributions are plotted in coordinates $X=\sqrt{T / T_{\mathrm{O}}}, Y=\sqrt{O / O_{\mathrm{T}}}$, where $T_{\mathrm{O}}=12(6 \sqrt{2}+7)^{-2}$ is the tetrahedricity index of a perfect quartoctahedron, and $O_{\mathrm{T}}=(\sqrt{2}-1)^{2} / 2$ is the octahedricity index of a perfect tetrahedron. These distributions display striking regularities. The distributions are placed in a region having straight lines as lower boundaries:

$$
Y=|1-X|
$$

(Fig. 12). At reduced temperatures, the distributions press close to a vertical plane constructed on the $Y=1-X$ line. This means that the basic types of DS lie exactly along this line. Lines (3) correspond to isopentakmons, ${ }^{*}$ i.e., to simplices with five identical edges of unit length and sixth edge of length $l$. When $l$ changes from 1 to $\sqrt{2}$, the point that represents the isopentakmon moves along the $Y=1-X$ line. As $l$ increases further from $\sqrt{2}$ to $\sqrt{3}$ (which is the limiting possible value), the point moves along the $Y=X-1$ line. From definitions (1) and (2) it follows that for the given tetrahedricity index $T$, the octahedricity index should always exceed the corresponding index of the isopentakmon with given $T$ (some violation of this rule visible in Fig. 12 occurs because of the finite size of the histogram cells). No mathematics, however, predicts that isopentakmons should prevail in the structure of a system, especially at low temperatures. This is the specific law of the structure of simple liquids.

Figure 12 displays some other characteristic lines. For a simplex with $n$ edges of length $l$ and $(6-n)$ edges of unity length, it was shown [7] that

$$
Y=\left[1-2\left(\frac{6-n}{5 n}\right)^{1 / 2} X+\left(1+\frac{6(n-1)}{n(6 \sqrt{2}+7)^{2}}\right) X^{2}\right]^{1 / 2},
$$

which follows from definitions (1) and (2). For isopentakmons, $n=1$; hence Eq. (4) transforms into Eq. (3). Figure 12 shows lines for $n=2,3$, and 4 . We can see that the boundaries of distributions at certain levels of probability lie along these lines. This means that Delaunay simplices tend to have identical edge lengths in liquids. The most probable (energetically

*This term was designed by Professor E. Anastassakis (Athens University) and means in Greek a figure with five identical edges. Our gratitude to Professor E. Anastassakis slipped from the text of footnote 19 in [7] because of some technical oversight. We are glad to correct this error here. 

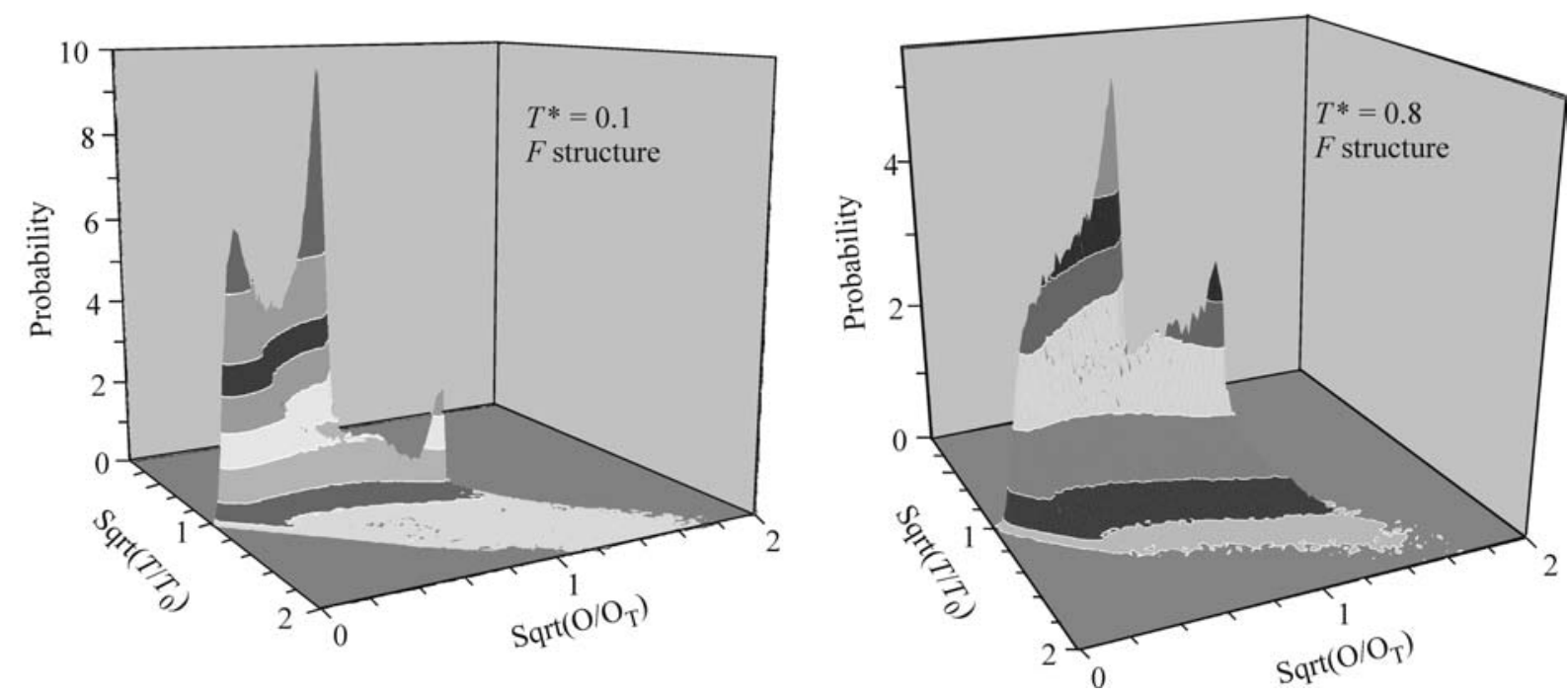

Fig. 11. Joint distribution of tetrahedricity and octahedricity of Delaunay simplices. $F$ structure, $\rho^{*}=0.85$. Left: $T^{*}=0.1$, right: $T^{*}=0.8$. Levels of coloring are identical in both pictures.
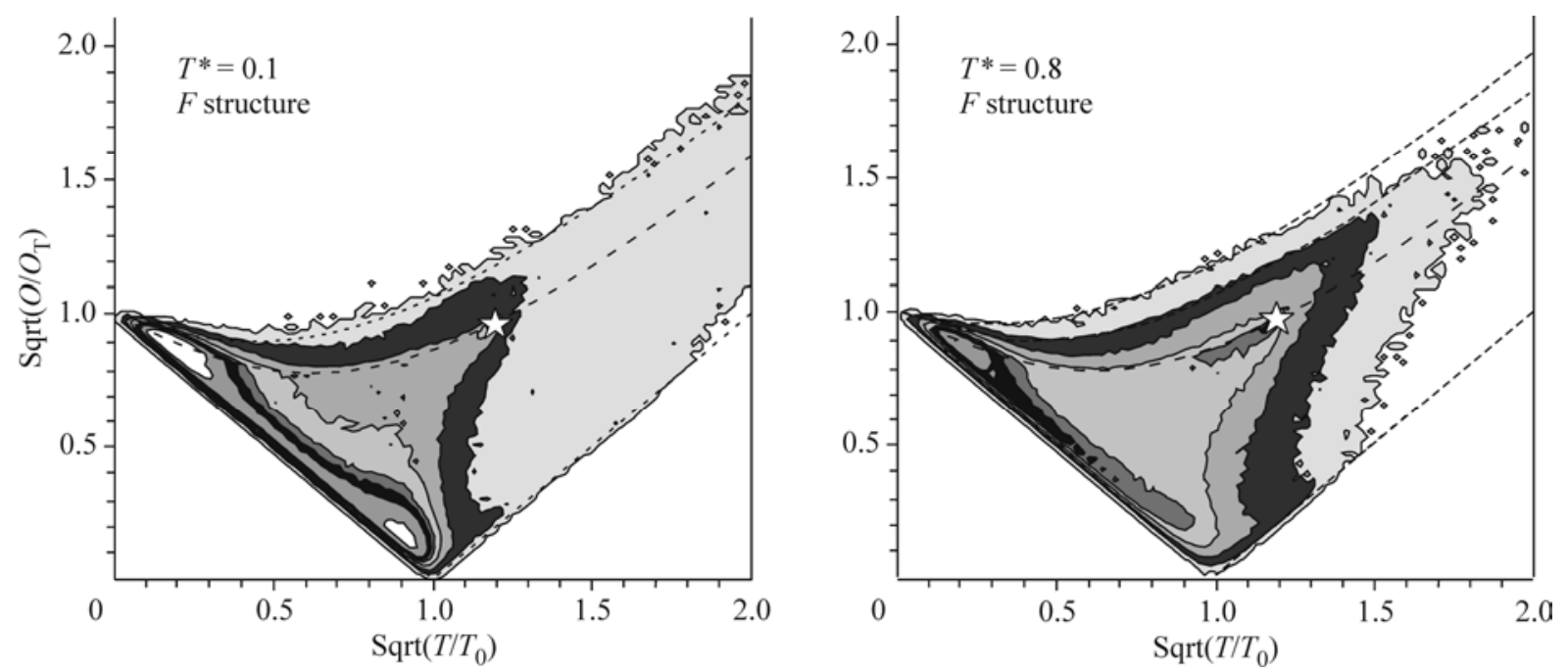

Fig. 12. Map of distribution levels shown in Fig. 11. The borders of the levels are identical in the two pictures. The lines corresponding to Eq. (4) are shown by the dotted line. From bottom to top: $n=1$ (isopentakmon line), $2,3,4$. The white asterisk shows the position of Kije simplices.

favorable) simplices are those in the form of nearly ideal tetrahedron $(n=0)$, as stated already by Bernal $[3,4]$. Simplices with five approximately identical edges (isopentakmons, quartoctahedra included) have lower probability, simplices with four edges $(n=2)$ have still lower probability, etc.

Three sharp peaks, most prominent at low temperatures (Fig. 11, left), are the most striking features of $(T, O)$ distributions. Two of them lie near the line of isopentakmons and correspond to good tetrahedra and good quartoctahedra (which are thus most outstanding among isopentakmons). The third peak corresponds to a Kije simplex (for a perfect Kije simplex, i.e., for a flat square, $X=1.188$ and $Y=0.963)$. When the temperature increases, the following transformations take place. The number of good tetrahedra and quartoctahedra decreases (see the numbers on the $Z$ axis, Fig. 11), but the peak of quartoctahedra goes down more quickly than the peak of tetrahedra, so that at high temperatures it does not rise above the ridge of isopentakmons (Fig. 11, right). Most good quartoctahedra and some good tetrahedra transform into simplices of intermediate forms (shown in yellow in Fig. 11). However, the sharp peak of Kije simplices always towers above the raising 


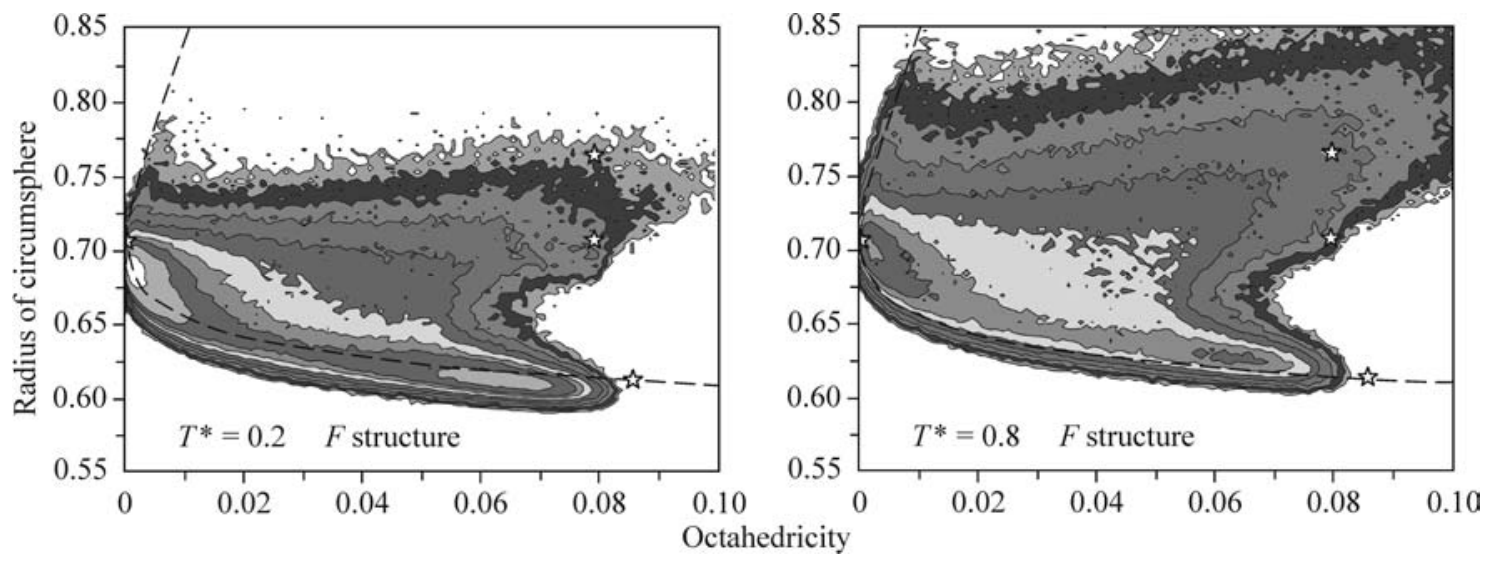

Fig. 13. Map of levels of the joint distribution of octahedricity and circumsphere radii. $\rho^{*}=0.85, F$ structure. Left: $T^{*}=0.2$, right: $T^{*}=0.8$. The line of isopentakmons is shown by a dashed line. Asterisks correspond to perfect figures (from bottom to top): tetrahedra, quartoctahedra (along the $Y$ axis), and Kije and anti-Kije simplices.

background of these intermediate simplices. Thus in liquids at high temperatures, good tetrahedra and Kije simplices are always visible against the background of the diffuse ridge of isopentakmons and intermediate simplices. Kije simplices give evidence of slightly deformed octahedral configurations of atoms. This means that octahedral configurations (though in small amounts) are also essential for optimum packing of tetrahedral simplices in addition to quartoctahedra.

Apart from $(T, O)$ distributions, one can construct other joint distributions of two characteristics of DS. Among these here we show only the $(R, O)$ distribution (Fig. 13). The pictures of this distribution are much richer than the distributions of octahedricity and circumsphere radius taken separately, which are merely projections of the former distribution. They demonstrate structural changes at different temperatures more clearly. At any temperatures, liquids have no ideal simplices. The probability of perfect tetrahedra is almost zero. Slightly deformed tetrahedra are present at maximum probability. Kije simplices are always present, but they are always found on the periphery of distributions. At higher temperatures, the distributions become less distinct and shift toward simplices conventionally called anti-Kije simplices. They belong to the class of DS with $n=2$; like Kije simplices, they have two edges $l=\sqrt{2}$ in length, but their long edges share a vertex, while in Kije simplices, they do not. Kije and anti-Kije simplices have identical values of $T$ and $O$ and thus are not differentiated on the $(T, O)$ diagrams (Figs. 11 and 12). In Fig. 13 one can also see that the ridge of the most probable DS lies slightly below the line of isopentakmons at $T^{*}=0.2$, and slightly above it at $T^{*}=0.8$. This is the consequence of removal of tensions in nonuniform systems (at $T^{*} \leq 0.3$ ), abruptly decreasing the distance between the nearest atoms (Fig. 4) and hence the radius of the sphere around DS (Fig. 10).

\section{PERCOLATION ANALYSIS}

The above-considered properties of Delaunay simplices speak nothing about the relative positions of these structural elements and hence cannot be used to represent the total structure of a substance. The ideology of percolation theory helps us to solve this problem. Delaunay simplices are "defined" on the Voronoi network: the centers of circumspheres around them are sites of this network (vertices of Voronoi polyhedra), and the edge between two sites shows that the simplices corresponding to these sites have a common face [5]. In other words, the Voronoi network defines the contiguity of the Delaunay simplices by faces. This allows one to investigate the structure of definitely shaped clusters consisting of adjacent DS. Let us separate on the Voronoi network only those Delaunay simplices whose shape deviates slightly from the perfect tetrahedron, for which the tetrahedricity index $T$ lies in the range from 0 to a certain boundary value $T_{\mathrm{b}}$. This procedure sets the coloring of the Voronoi network according to the tetrahedricity of the DS ( $T$ coloring). If the fraction of the separated 
TABLE 1. Percolation Characteristics for a Lennard-Jones System $\left(N=100,000, \rho^{*}=0.85\right)$

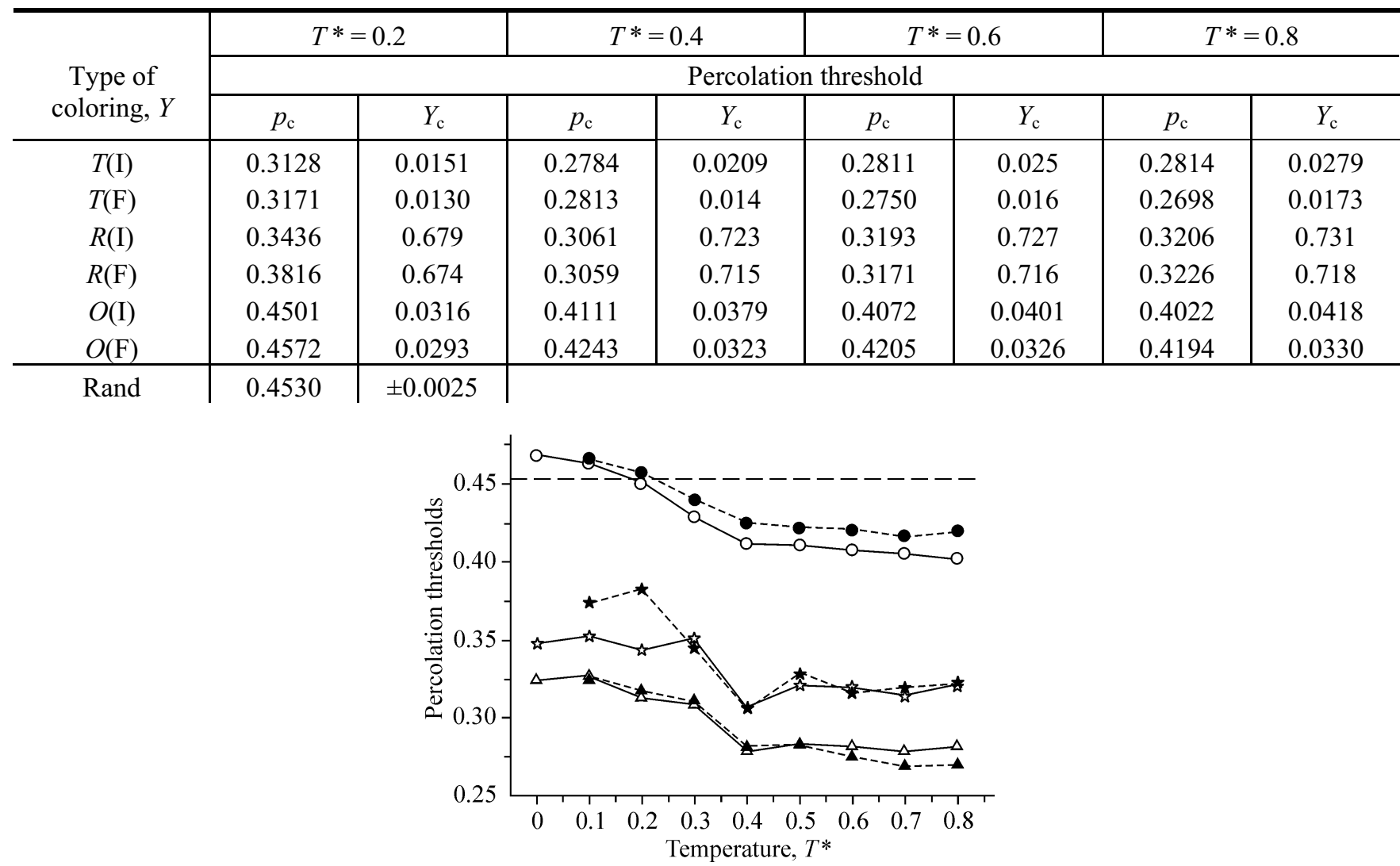

Fig. 14. Temperature behavior of percolation thresholds for different colorings on the same Voronoi network of the model, $\rho^{*}=0.85$. Triangles: $T$ coloring, circles: $O$ coloring, asterisks: $R$ coloring. Empty symbols: $I$ structure; full symbols: $F$ structure. The horizontal dashed line shows the percolation threshold for random coloring.

(colored) simplices $p$ is small, then only isolated clusters of adjacent DS are colored on the network. As $p$ increases, the clusters unite, and at a certain critical value of $p_{\mathrm{c}}$ (and the corresponding value of the boundary $T_{\mathrm{c}}$ ) an "infinite" cluster appears; i.e., face-sharing DS extend from one border of the model to another. The value of $p_{\mathrm{c}}$ defines the percolation threshold for the Voronoi network colored according to tetrahedricity. Similarly, one can determine the percolation threshold by coloring the same network using the octahedricity criterion ( $O$ coloring), by gradually increasing the boundary index $O_{b}$ of simplices and hence the fraction of the colored sites. The network may also be colored using the radius of the circumspheres ( $R$ coloring), starting from the largest radii and gradually reducing $R$ until an infinite cluster has appeared. This type of coloring provides information on the spatial arrangement of regions with decreased local density.

The values of percolation thresholds obtained for different types of coloring are presented in Table 1 and Fig. 14. For coloring by radius and especially by tetrahedricity, percolation thresholds differ significantly from those for random coloring (Rand) of the same network. This means that DS in the form of good tetrahedra and DS with large radii of circumspheres are located not randomly in space. The same is also valid for DS shaped like good quartoctahedra, although for $O$ coloring the thresholds differ less significantly from $p_{\text {c }}$ for random coloring. For all types of coloring, percolation thresholds change but slightly with temperature, especially in the region of uniform states at $T^{*} \geq 0.4$ (Fig. 14). For models with $\rho^{*}=0.7$ and 0.9 , the thresholds (in the case of $T$ and $O$ coloring) and their temperature dependence proved to be the 
TABLE 2. Percolation Characteristics for Different Models of Lennard-Jones System

\begin{tabular}{|c|c|c|c|c|c|c|c|c|}
\hline \multirow[t]{2}{*}{$\begin{array}{l}\text { Type of } \\
\text { coloring, } Y\end{array}$} & \multicolumn{2}{|c|}{$\begin{array}{c}N=108 \\
\rho^{*}=0.9 \\
T^{*}=0.719\end{array}$} & \multicolumn{2}{|c|}{$\begin{array}{c}N=100.000 \\
\rho^{*}=0.9 \\
T^{*}=0.8\end{array}$} & \multicolumn{2}{|c|}{$\begin{array}{c}N=8000 \\
\rho^{*}=1.0 \\
T^{*}=0\end{array}$} & \multicolumn{2}{|c|}{$\begin{array}{c}N=8000 \\
\rho^{*}=0.8 \\
T^{*}=0\end{array}$} \\
\hline & $\begin{array}{c}\text { Percolation } \\
\text { threshold } \\
\quad p_{\mathrm{c} \pm \sigma}\end{array}$ & $Y_{\mathrm{c}}$ & $p_{\mathrm{c}}$ & $Y_{\mathrm{c}}$ & $p_{\mathrm{c}}$ & $Y_{\mathrm{c}}$ & $p_{\mathrm{c}}$ & $Y_{\mathrm{c}}$ \\
\hline$T(\mathrm{I})$ & $0.311 \pm 0.022$ & 0.026 & 0.2805 & 0.0246 & & & & \\
\hline$T(\mathrm{~F})$ & $0.287 \pm 0.029$ & 0.018 & 0.2778 & 0.0158 & 0.2930 & 0.014 & 0.2736 & 0.018 \\
\hline$R(\mathrm{I})$ & $0.340 \pm 0.035$ & 0.705 & 0.3324 & 0.709 & & & & \\
\hline$R(\mathrm{~F})$ & $0.351 \pm 0.049$ & 0.695 & 0.3384 & 0.698 & 0.3756 & 0.750 & 0.2183 & 0.866 \\
\hline$O(\mathrm{I})$ & $0.429 \pm 0.024$ & 0.037 & 0.4089 & 0.0398 & & & & \\
\hline$O(\mathrm{~F})$ & $0.423 \pm 0.038$ & 0.031 & 0.4254 & 0.3222 & 0.4320 & 0.030 & 0.4070 & 0.034 \\
\hline Rand & \multicolumn{2}{|c|}{$0.456 \pm 0.028$} & \multicolumn{2}{|c|}{$0.4530 \pm 0.0025$} & \multicolumn{2}{|c|}{$0.4527 \pm 0.0071$} & \multicolumn{2}{|c|}{$0.4550 \pm 0.0075$} \\
\hline
\end{tabular}

same as for the model with $\rho^{*}=0.85$. For all types of coloring, the percolation thresholds for $I$ and $F$ structures are practically identical (Fig. 14). The contiguity of Delaunay simplices, therefore, is not violated from $I$ to $F$ structures, despite changes in their metrical properties $\left(Y_{\mathrm{c}}\right.$, Table 1). Thus we can see once again that the proper structure of a liquid (whose particular case is the $F$ structure) retains the true structural regularities, disguised by thermal chaos in the $I$ structure.

Table 2 summarizes percolation characteristics for models under study. When the number of particles increased by three orders of magnitude, the accuracy in determining the percolation threshold increased by only one order of magnitude. For random coloring, the percolation threshold was identical for networks at all temperatures and also for $I$ and $F$ structures: $p_{\mathrm{c}}=0.4530 \pm 0.0025$. This is very close to $p_{\mathrm{c}}=0.4301$ for random coloring of the diamond lattice [16], but differs from it beyond the limits of the error. Many authors believe that the percolation threshold for site coloring is determined exclusively by the coordination number of the lattice $[15,16]$. However, the Voronoi network and the diamond lattice have the same coordination number, 4 . Therefore the above values show that the threshold also depends on the network topology.

Unlike the percolation threshold, the fraction of the sites belonging to the infinite cluster, $P_{\infty}$, strongly fluctuates from one realization of random coloring to another, varying by more than one order of magnitude. Nevertheless, the ratio of the number of bonds to the number of sites in the infinite cluster fluctuates very slightly. The constancy of this ratio for large fluctuations of $P_{\infty}$ means that the branching of the infinite cluster remains the same: just as a tree consists of different numbers of branches, so an infinite cluster consists of different numbers of equally arranged blocks.

\section{TOTAL STRUCTURE}

The general view of the structure of models at long distances is presented on the pictures of colored Voronoi networks. Figure 15 shows the patterns for different types of network coloring in a model of 8000 atoms investigated in [11]. For large models (100,000 particles), such pictures cannot be drawn on paper because of the large number of details. Computer models, however, indicate that the arrangement of large models is similar to that of small ones. Similar patterns were also obtained for models of 109 particles [6,8]. Figure 15 presents only the backbones of the clusters, i.e., bond rings and edges to connect them (having no "dead ends," i.e., edge chains not leading to rings). Thus to represent a full picture of colored clusters, one should bear in mind that the rings shown in Fig. 15 are built into branched chains, and clusters without rings are present in addition to these.

The most impressive picture is offered by $T$ coloring (imaging the centers of adjacent Delaunay simplices, approaching the ideal tetrahedron in shape). Figure $15 a$ shows that the backbones of clusters of this kind consist only of fivemembered rings. Similar fine pictures of five-membered rings are also visible in models of molten close-packed metals [27]. These rings correspond to five almost perfect tetrahedra united into a bipyramid (decahedron) in which they share two faces 


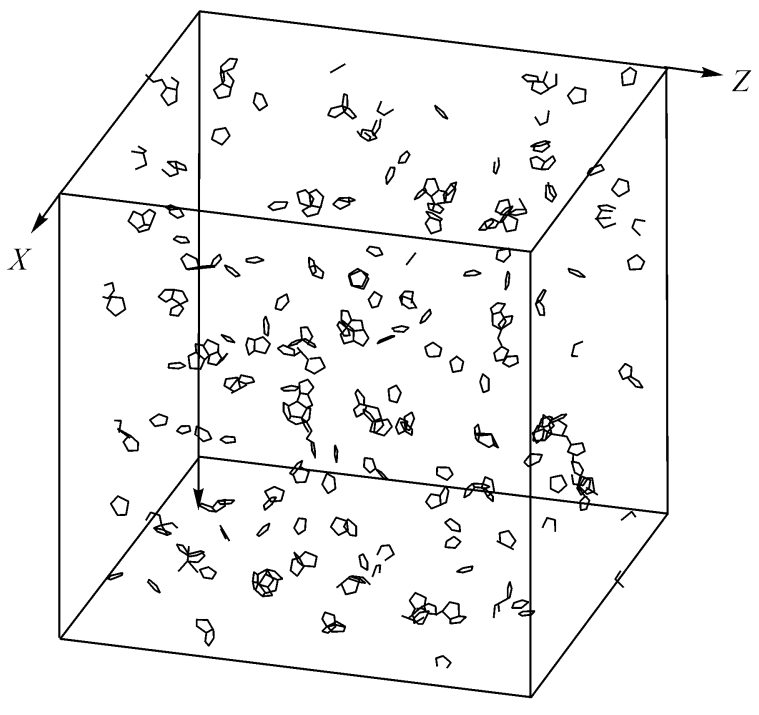

$b$

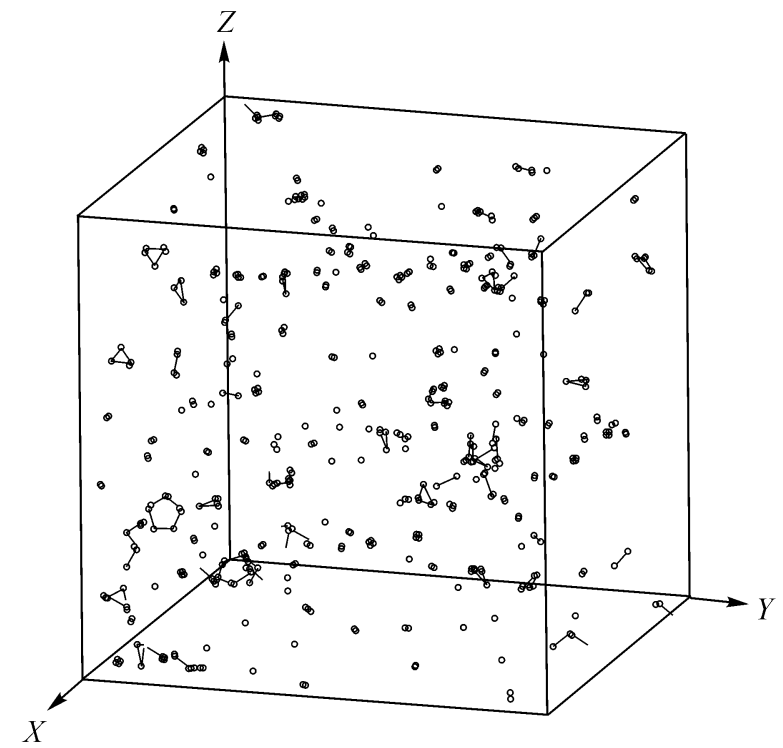

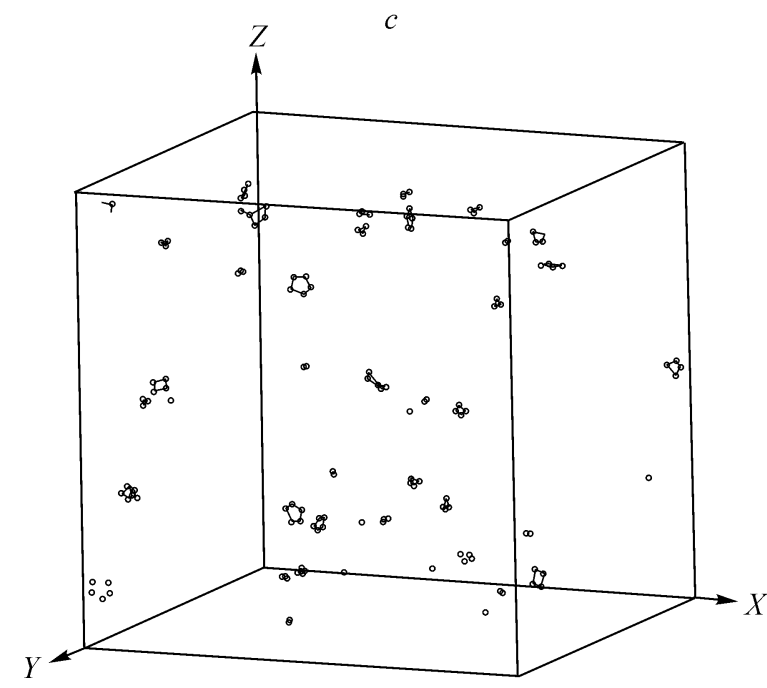

Fig. 15. Various types of coloring of the Voronoi network for the model with 8000 atoms $\left(\rho^{*}=1.0\right)$. The total number of Delaunay simplices (network sites) is 48,647. The backbones of the colored clusters are shown: $a-$ $T$ coloring; DS with $T<T_{\mathrm{b}}=0.005$ are colored; $12.7 \%$ of all sites (2415 clusters) are colored; $2.15 \%$ sites remained in the backbones (142 clusters); $b-O$ coloring; $O_{\mathrm{b}}<0.01,15.9 \%$ of all sites (3571 clusters) are colored; $1.92 \%$ sites remained in the backbones (185 clusters); $c$ - random coloring; $20 \%$ of all sites (6003 clusters) are colored; $0.35 \%$ sites (44 clusters) remained in the backbones.

and one edge $[5,6,8]$. The rings are sometimes isolated; as the fraction of colored sites increases, they are linked by bonds and increasingly condensed. The remaining Delaunay simplices, shaped like good tetrahedra (which, as we have seen, represent the majority of all DS), are organized into clusters in the form of branched polytetrahedral chains (without rings); these compose the major part of all clusters: coloring in Fig. $15 a$ gave 2415 clusters, of which only 142 have backbones. The presence of five-membered rings of good tetrahedra is the most striking property of the total structure of simple liquids. Bernal explained this as follows [3,4]: a packing of five tetrahedra has the greatest local density and hence is the most favorable configuration. Polytetrahedral configurations of fivefold symmetry are ruled out in crystals, but are the basic motif of the total structure of simple liquids.

The picture of coloring by octahedricity is quite different (Fig. 15b). Five-membered rings occur randomly, most 


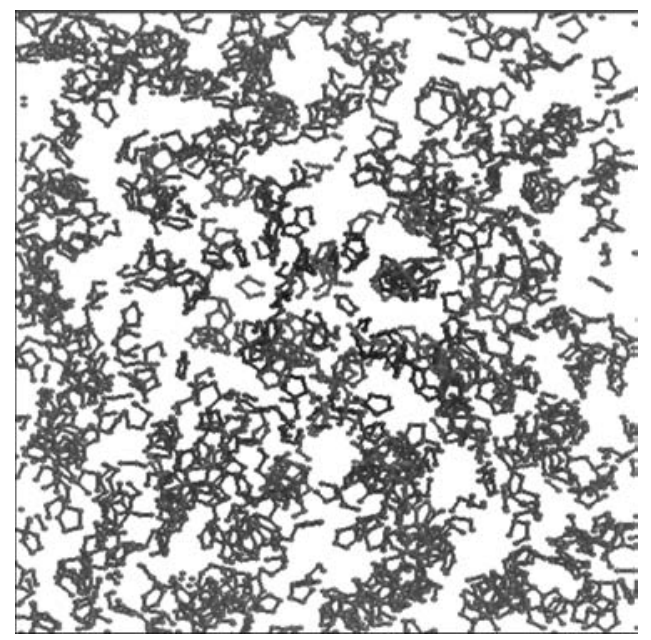

Fig. 16. Coloring of the Voronoi network by tetrahedricity for the model of 8000 atoms. $T_{\mathrm{b}}=0.01$. Cluster backbones are shown.

backbones being four-membered rings with very short edges indistinguishable on the scale of the figure. These rings correspond to four quartoctahedra associated into a weakly deformed full octahedron. Although the octahedral configurations are present in extremely small amounts, these are essential elements of atomic packing in simple liquids that give rise to Kije simplices, well visible in Figs. 11 and 12. Random coloring of the same Voronoi network bears no relationship to $T$ or $O$ coloring (Fig. 15c). It unambiguously shows that good tetrahedra and quartoctahedra are arranged nonrandomly in space.

Nonrandom arrangement of the basic structural elements in simple liquids entails their structural heterogeneity. This is demonstrated here by $T$ coloring. Figure 16 shows the whole model box as a "transparent" figure. Colored backbones are crowded into regions separated by "empty space" regions consisting of simplices of another (uncolored) form.

\section{CONCLUSIONS}

The choice of Delaunay simplices as structural elements allows one to reveal interesting properties of the structure of simple liquids. At low temperatures, most Delaunay simplices are isopentakmons, i.e., tetrahedra with five identical edges. Among isopentakmons one can distinguish simplices whose shape approaches that of a perfect tetrahedron and quartoctahedron (good tetrahedra and good quartoctahedra); these forms prevail in the structure of liquids at all temperatures. The third type of simplex is a Kije simplex (slightly deformed square); this is generally present in small amounts, but is an indispensable element of structure. At elevated temperatures the fraction of simplices of intermediate forms increases, but the above-mentioned three basic types of Delaunay simplex always persist in the structure. The ratio of the number of good tetrahedra to the number of good quartoctahedra in liquid exceeds by far the same ratio in crystal, indicating that liquids have different arrangement than crystals. Simplices in the form of good tetrahedra and quartoctahedra are located not randomly in space, which leads to structural heterogeneity of the model. Good tetrahedra form clusters in the form of branched chains having built-in five-membered rings; they span the entire system even when colored good tetrahedra are present in small amounts. The structure of simple liquids has local areas of fivefold symmetry; this is probably the most striking difference between liquid and crystal structures.

\section{REFERENCES}

1. S. R. Elliott, Physics of Amorphous Materials, Longman, London (1990).

2. J. M. Ziman, Models of Disorder, Cambridge University, Cambridge (1979). 
3. J. D. Bernal, Nature, 183, 141 (1959).

4. J. D. Bernal, Proc. R. Soc. A, 280, 299-322 (1964).

5. N. N. Medvedev, Voronoi-Delaunay Method in Structural Studies of Noncrystalline Systems [in Russian], Siberian Division, Russian Academy of Sciences, Novosibirsk (2000).

6. N. N. Medvedev, V. P. Voloshin, and Yu. I. Naberukhin, Zh. Strukt. Khim., 30, No. 2, 98-105 (1989).

7. V. P. Voloshin, Yu. I. Naberukhin, and N. N. Medvedev, Mol. Simul., 4, 209-227 (1989).

8. Yu. I. Naberukhin, V. P. Voloshin, and N. N. Medvedev, Mol. Phys., 73, 917-936 (1991).

9. V. P. Voloshin and Yu. I. Naberukhin, J. Phys.: Condens. Matter., 5, 5685-5700 (1993).

10. V. P. Voloshin and Yu. I. Naberukhin, Zh. Strukt. Khim., 38, No. 1, 78-88 (1997).

11. V. P. Voloshin and Yu. I. Naberukhin, ibid., 41, No. 5, 1005-1012 (2000).

12. V. A. Luchnikov, N. N. Medvedev, Yu. I. Naberukhin, and H. R. Schober, Phys. Rev. B, 62, No. 5, 3181-3189 (2000).

13. V. P. Voloshin and Yu. I. Naberukhin, Zh. Strukt. Khim., 46, No. 2, 273-283 (2005).

14. G. B. Bokii, Kristallokhimiya [in Russian], Nauka, Moscow (1971).

15. A. L. Éfros, Physics and Geometry of Disorder [in Russian], Nauka, Moscow (1982).

16. Yu. Yu. Tarasevich, Percolation: Theory, Applications, Algorithms [in Russian], URSS, Moscow (2002).

17. P. M. Zorky, L. V. Lanshina, and E. Yu. Korableva, Zh. Strukt. Khim., 35, No. 2, 121-126 (1994).

18. P. M. Zorky, Ross. Khim. Zh. (Zh. Ross. Khim. Ova D. I. Mendeleeva), 45, No. 2, 3-10 (2001).

19. J. P. Hansen and L. Verlet, Phys. Rev., 184, No. 1, 151-161 (1969).

20. F. H. Stillinger and T. A. Weber, Phys. Rev. A, 25, 978-989 (1982).

21. F. H. Stillinger and T. A. Weber, J. Chem. Phys., 81, No. 11, 5095-5103 (1984).

22. F. H. Stillinger and T. A. Weber, Science, 225, 983-989 (1984).

23. O. S. Subbotin and V. R. Belosludov, Zh. Strukt. Khim., 47, Supplement, S65-S69 (2006).

24. Yu. I. Naberukhin, V. P. Voloshin, and N. N. Medvedev, Rasplavy, 1, No. 2, 71-77 (1987).

25. G. G. Malenkov, A. V. Teplukhin, and V. I. Poltev, Zh. Strukt. Khim., 30, No. 4, 89-97 (1989).

26. R. Zallen, The Physics of Amorphous Solids, Wiley, New York (1983).

27. A. S. Roik, V. P. Kazimirov, and V. É. Sokolskii, Zh. Strukt. Khim., 45, No. 4, 682-691 (2004). 